\section{ORIGINAL RESEARCH}

J.Y. Chueh

A.K. Wakhloo

G.H. Hendricks

C.F. Silva

J.P. Weaver

M.J. Gounis

\title{
Mechanical Characterization of Thromboemboli in Acute Ischemic Stroke and Laboratory Embolus Analogs
}

BACKGROUND AND PURPOSE: Mechanical behavior of the thromboembolus is one of the key factors that determine the efficacy of thrombectomy devices for revascularization in AIS. We characterized the mechanical properties and composition of thromboemboli from clinical cases and compared them with commonly used EAs.

MATERIALS AND METHODS: Thromboemboli were obtained from patients with AIS by using aspiration devices and from carotid atherosclerotic plaques harvested during endarterectomy. In the laboratory, common EAs were created by varying blood donor species (human, porcine, and bovine), thrombin concentration, and presence of barium sulfate. Stiffness and elasticity of the specimens were measured with DMA. Scanning electron microscopy and histology were used to investigate the ultrastructure and composition of all specimens.

RESULTS: Red thromboemboli from patients composed mainly of fibrin and erythrocytes were much softer than the calcified and cholesterol-rich material. Of the EAs created in the laboratory, those made from bovine blood presented the highest stiffness that was independent of thrombin concentration. Addition of thrombin increased the stiffness and elasticity of human and porcine $\mathrm{EAs}(P<.05)$. The presence of barium sulfate significantly reduced the elasticity of all EAs $(P<.05)$.

CONCLUSIONS: Endovascular device testing and development requires realistic EAs. The stiffness and elasticity of the cerebral thromboemboli analyzed in this study were closely matched by recalcified porcine EAs and thrombin-induced human EAs. Stiffness of the thrombus extracted from carotid endarterectomy specimens was similar with that of the thrombin-induced bovine and porcine EAs.

\begin{abstract}
ABBREVIATIONS: $\mathrm{ACD}=$ anticoagulant citrate dextrose; $\mathrm{AIS}=$ acute ischemic stroke; $\mathrm{CEA}=$ carotid endarterectomy; $\mathrm{DMA}=$ dynamic mechanical analyzer; $\mathrm{E}_{\text {intitial strain-end strain }}=$ secant modulus in a given strain range (initial strain to end strain); $E A=$ embolus analog; $E D S=$ energy dispersive $\mathrm{x}$-ray spectroscopy; H\&E = hematoxylin-eosin; ICA = internal carotid artery; $\mathrm{MCA}=$ middle cerebral artery; MSB = Martius scarlet blue; NIHU = National Institutes of Health unit; $\mathrm{S}-\mathrm{S}=$ stress-strain; $\mathrm{SEM}=$ scanning electron microscopy
\end{abstract}

W ith growing experience of penumbral imaging for patient selection and newer, more effective mechanical revascularization devices, alternative endovascular therapy for AIS has become a viable treatment option. ${ }^{1}$ There are presently 2 devices available in the United States for thrombectomy in AIS, and many other technologies are under evaluation. These devices produce recanalization in $50 \%-82 \%$ of

Received September 21, 2010; accepted after revision November 18.

From the Department of Radiology (J.Y.C., A.K.W., C.F.S., M.J.G.), New England Center for Stroke Research, University of Massachusetts Medical School, Worcester, Massachusetts; Department of Cell Biology (G.H.H.), University of Massachusetts Medical School, Worcester, Massachusetts; and Department of Surgery (J.P.W.), Division of Neurosurgery University of Massachusetts Medical School, Worcester, Massachusetts.

This work was supported by grant 1R21EB007767 from the National Institutes of Health National Institute of Biomedical Imaging and Bioengineering. The contents are solely the responsibility of the authors and do not necessarily represent the official views of the National Institutes of Health

Previously presented as posters at: 48th Annual Meeting of the American Society of Neuroradiology, May 15-20, 2010, Boston, Massachusetts; Summer Bioengineering Conference, June 16-19, 2010, Naples, Florida; and 7th Annual Meeting of the Society for Neurointerventional Surgery, July 26-29, 2010, Carlsbad, California.

Please address correspondence to Matthew J. Gounis, PhD, Asst Professor, Department of Radiology, Director, New England Center for Stroke Research, University of Massachusetts, 55 Lake Ave N, SA-107R, Worcester, MA 01655; e-mail: matt.gounis@umassmed.edu

Indicates open access to non-subscribers at www.ajnr.org

Indicates article with supplemental on-line table.

DOI 10.3174/ajnr.A2485 cases. ${ }^{2,3}$ Currently, it is poorly understood what variables predict successful recanalization. Biomechanical characterization of the thromboembolus is helpful in device development.

Preclinical evaluation of thrombectomy devices measures efficacy and safety in animal or in vitro vascular occlusion models. ${ }^{4-7}$ Common end points of these studies are the number of thrombectomy attempts, the amount of EAs removed from the occlusion site, and the risk of distal embolic shower. ${ }^{5,6,8-10}$ All of these metrics are significantly affected by the mechanical properties of the EAs, an often overlooked component of the testing system. In the literature, there are many protocols to manufacture EAs, and common variables include the donor species, concentration of thrombin, and radiopaque additive such as barium sulfate. ${ }^{6,9-13}$ Variation of hematologic values among different donors along with the $\mathrm{pH}$ and ionic strength of the clotting environment result in different structure and composition of the EAs. ${ }^{14-18}$ This leads to the hypothesis that commonly used EAs have different mechanical properties. Two recent studies reported the composition of emboli retrieved from the stroke patients, ${ }^{19,20}$ and substantiated the structural and compositional variations between the in vivo emboli and in vitro EAs. ${ }^{21}$ However, the mechanical properties of EAs and how they relate to thromboemboli from patients has not been studied.

The goal of this study is to compare the mechanical prop- 


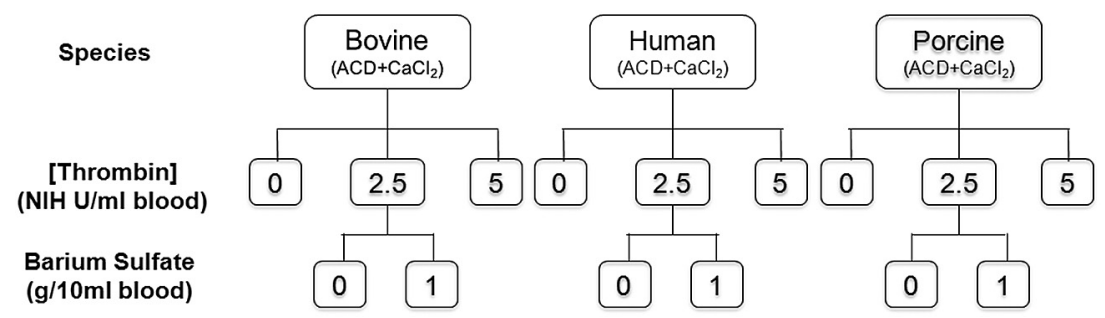

Fig 1. Preparation of EAs. Three variables of in vitro clotting include species, thrombin concentration, and addition of barium sulfate.

erties (stiffness and elasticity) and composition of thromboemboli from patients with EAs made in the laboratory.

\section{Materials and Methods}

\section{Collection of Thrombi from Patients}

All human specimens were collected with approval from our Institutional Review Board. Nine thromboemboli were obtained from the ICA or MCA of 4 stroke patients by aspiration and were prepared for material characterization. Because debris from atherosclerotic plaques and atrial fibrillation are well documented as important sources of emboli to cause AIS, ${ }^{22,23} 26$ atherosclerotic plaques obtained during CEA and 1 atrial appendage were collected. Materials loosely attached to the luminal surface of the plaques and red thrombi found under the fibrous cap were considered potential sources of cerebral emboli and therefore harvested. After careful examination under the microscope, 13 thromboemboli in total were extracted from 7 asymptomatic and 1 symptomatic plaque (8 patients). Clinical data of the patients and details of thromboemboli are provided in the On-line Table, where the tested specimens were numbered by the procedure type (C, carotid endarterectomy; A, aspiration in acute ischemic stroke) followed by an integer denoting the order in which the embolus was obtained.

\section{Preparation of EAs}

The effects of thrombin and barium sulfate on the stiffness and elasticity of the bovine, porcine, and human EAs were studied. The donor species, concentration of thrombin $(0,2.5$, and $5 \mathrm{NIHU} / \mathrm{mL}$ blood), and presence of barium sulfate $(0$ and $1 \mathrm{~g} / 10 \mathrm{~mL}$ blood $)$ were selected based on those commonly used for EA preparation in previous work (Fig 1). ${ }^{5,6,9-12}$

Without the presence of thrombin, spontaneous coagulation was initiated by mixing bovine, porcine, or human whole blood/ACD mixture (10:1) and calcium chloride $(97 \mathrm{mmol} / \mathrm{L})$ at a 5:1 ratio. Based on the species divergence of structure and function in fibrinogen ${ }^{18}$ and specificity of the thrombin-fibrinogen interaction, ${ }^{17}$ bovine thrombin (T-7513; Sigma, St. Louis, Missouri) was used to initiate fibrin formation in bovine and porcine EAs, whereas human thrombin (T-6884; Sigma) was used in human blood samples. Thrombininduced clotting was conducted by simultaneously injecting whole blood/ACD mixture (10:1) and calcium chloride $(97 \mathrm{mmol} / \mathrm{L}) /$ thrombin solution (1 NIHU thrombin/4 $\mu \mathrm{L}$ solution) into silicone tubing $(6.35 \mathrm{~mm}$ inner diameter) to give blood mixtures with final thrombin concentration of 2.5 and $5 \mathrm{NIHU} / \mathrm{mL}$ blood. Before injection, silicone tubing was rinsed with $70 \%$ alcohol followed by $0.9 \%$ saline. The radiopaque EA was created by adding $1 \mathrm{~g}$ of barium sulfate into the $10-\mathrm{mL}$ blood mixture that had a thrombin concentration of $2.5 \mathrm{NIHU} / \mathrm{mL}$ blood. ${ }^{12}$ All EAs were aged in saline at room temperature for 1 day before characterization.

\section{Mechanical Characterization}

DMA (Q800; TA Instruments, New Castle, Delaware), which has a force resolution of $0.00001 \mathrm{~N}$ and a strain resolution of $1 \mathrm{~nm}$, was used to explore the stiffness and elasticity of the thromboemboli and EAs. Stiffness is a material characteristic that describes the resistance to deformation under an applied load. Elasticity, in contrast, is the ability of a material to return to its original shape after being deformed. All mechanical examinations were conducted by using a submersion compression clamp within saline at $37^{\circ} \mathrm{C}$. The EAs were cut to have a height of $2 \mathrm{~mm}$, and a caliper having a resolution of $0.1 \mathrm{~mm}$ was used to measure the diameter of the EA. The specimens retrieved from patients had an irregular shape and were carefully trimmed under a microscope to have a height between 1 and $2 \mathrm{~mm}$. To prevent test material (thromboemboli or EAs) from slipping, 220-grit sandpaper was adhered to the compression disk.

In the controlled force mode, the test materials (a minimal sample size of $n=5$ for each group) were first subjected to a preload force of $0.0001 \mathrm{~N}$, followed by a compression force ramp from 0.0001 to $15 \mathrm{~N}$ at a rate of $0.5 \mathrm{~N} / \mathrm{min}$. The deformation of the test material caused by the compression stress was presented by the engineering S-S curve. Onset point, which is the intersection of the initial tangent line with the final tangent line, was recorded to determine the strain at which a change in the slope of the S-S curve occurred. Stress is defined as force over surface area, and strain refers to deformation (percent change in sample height). To quantitatively describe the deformation of the test material under the compression force that simulates the large strain induced by the thrombectomy devices during treatment, stress variation over a range of strain (the slope of the S-S curve) was calculated. The resulting $\mathrm{E}_{\text {intitial strain-end strain }}$ is an indication of material stiffness. Area under the S-S curve, which represents the energy required to deform the material, also was calculated.

In the stress-relaxation mode, the test materials were subjected to an initial strain of $60 \%$ for 5 minutes, followed by a recovery period of 15 minutes. The strain recovery (percentage), a measure of material elasticity, was acquired.

\section{Histologic Assessment}

Thromboemboli and EAs were fixed in a 10\% buffered formalin solution for 48 hours. Specimens were then embedded in paraffin wax and cut into $5-\mu \mathrm{m}$ sections. Sections were dewaxed and hydrated with distilled water in preparation for a modified version of the MSB method for staining fibrin, collagen, and erythrocytes. ${ }^{24}$ Alternating sections were stained with H\&E.

\section{SEM}

Thromboemboli and EAs were fixed with 2.5\% glutaraldehyde and dehydrated in a series of ethanol concentrations up to $100 \%$. To observe the interior of the specimens, they were frozen in liquid nitrogen 
and fractured. Samples were critical-point-dried, mounted, and sputter-coated with iridium for SEM observation. EDS was performed on 2 samples.

\section{Statistical Analysis}

Data were presented as mean \pm standard error of the mean. The unpaired $t$ test was performed to compare the means of the noncalcified plaque materials and cerebral emboli retrieved from the AIS patients. One-way analysis of variance followed by a Dunnett or Tukey posttest was used to determine significance between patients' thromboemboli (control) and EAs or between the EAs, respectively. Statistical significance was set at $P<.05$. Statistical analysis was performed by using Prism software (GraphPad Software, San Diego, California).

\section{Results}

\section{Composition and Structure of Patients' Thromboemboli}

From September 2009 to May 2010, 26 atherosclerotic plaques, 1 atrial appendage, and 9 cerebral thromboemboli were collected from 31 patients. Thirteen thrombi were harvested from 8 plaques collected at CEA under a microscope; no thromboemboli were found in the atrial appendage. Of the 13 specimens obtained from the CEA, 7 where adherent to the lumen of the vessel, and the remaining specimens were found under the fibrous cap. In total, 22 clinical specimens from 12 patients (mean age, 70 years) with an average thickness of $1.43 \pm 0.11 \mathrm{~mm}$ and surface area of $6.22 \pm 0.97 \mathrm{~mm}^{2}$ were characterized (On-line Table).

Figure 2 illustrates 2 representative patients who underwent thromboemboli extraction by aspiration or surgical removal. Based on composition and mechanical properties, we found that thromboemboli harvested from the patients can be classified into 3 categories: 1) calcified, 2) aged, and 3) red thromboemboli. The calcified thromboemboli (specimen numbers C2 and C7, On-line Table) had large amounts of calcium-phosphate apatite (Fig 3A). Aged thromboemboli having a dark reddish brown color and loosely attached to the vessel lumen had highly compact structures that consisted of old fibrin, adhesive proteins, connective tissue, and other cells, such as erythrocytes and platelets (Fig $3 B,-C$ ). In contrast, red thromboemboli (Fig 3D, $-F$ ) were composed of an aggregation of erythrocytes (yellow in Fig 3D) trapped in the fibrin network and leukocytes dispersed throughout the thromboembolus (Fig 3E). At higher magnification, a red thromboembolus from an AIS patient showed that the cellular components were arranged in a specific lamellar pattern (Fig $3 F)$.

\section{Biomechanics of Patient Thromboemboli}

The S-S curves of the calcified thromboemboli showed an early onset point $(42.9 \pm 8.0 \%)$ and small strain under a $15-\mathrm{N}$ load (the highest force experienced by the sample), compared with the aged and red thromboemboli (Fig 4). High stress was required to cause $45 \%$ strain in the calcified thromboemboli compared with other specimens. Aged thromboemboli collected during CEA had a later onset point $(85.4 \pm 0.9 \%)$ and larger strain under a $15-\mathrm{N}$ force (Fig 4). The stiffness of the aged thromboemboli $\left(\mathrm{E}_{0 \%-45 \%}=0.17 \pm 0.039 \mathrm{MPa}\right)$ was be- tween that of the calcified $\left(\mathrm{E}_{0 \%-45 \%}=0.63 \pm 0.38 \mathrm{MPa}\right)$ and red $\left(\mathrm{E}_{0 \%-45 \%}=0.026 \pm 0.0026 \mathrm{MPa}\right)$ samples. The red thromboemboli had the latest onset point (91.29 $\pm 0.82 \%)$ and largest strain upon loading with $15 \mathrm{~N}$.

The average $\mathrm{E}_{0 \%-75 \%}$ and $\mathrm{E}_{75 \%-95 \%}$ values of the thromboemboli retrieved from the AIS patients were $0.04 \pm 0.01$ and $0.43 \pm 0.06 \mathrm{MPa}$, respectively, whereas those of the noncalcified thromboemboli obtained from the CEA plaques were $0.11 \pm 0.037$ and $1.60 \pm 0.50 \mathrm{MPa}$, respectively. It should be noted that large variations in $\mathrm{E}_{0 \%-75 \%}$ and $\mathrm{E}_{75 \%-95 \%}$ were seen in the CEA thromboemboli (Fig 5A) reflecting the inhomogeneity of the structure and composition of these materials. Stress relaxation tests, only performed in the red thromboemboli of AIS patients, revealed the average strain recovery of $32.9 \pm 2.3 \%$ (Fig $5 B$ ). It was not possible to perform the stress relaxation measurements in the calcified material due to the high force required to reach $60 \%$ strain that could have damaged the equipment. Furthermore, the strain recovery of the aged thromboemboli was immeasurable due to fragmentation.

\section{Composition, Structure, and Biomechanics of EAs}

A layer of attenuated fibrin mesh was formed on the outer surface of the in vitro EA against the silicone tubing (Fig $6 A$, $-C)$, and the EAs were mainly composed of homogeneously dispersed erythrocytes with several interspersed fibrin bands (Fig $6 B,-D$ ). The average onset point of these erythrocyte-rich EAs analyzed under DMA-controlled force mode $(n=65)$ was $89.1 \pm 0.6 \%$.

Overall, bovine EAs showed the highest stiffness followed by porcine and human EAs (Fig 7). Without addition of thrombin, $\mathrm{E}_{0 \%-75 \%}$ and $\mathrm{E}_{75 \%-95 \%}$ of bovine EAs were statistically higher than those of porcine and human EAs $(P<.05)$. However, there was no significant difference in stiffness between EAs made from porcine and human blood (Fig 7A, -B). Increases in stiffness and elasticity of human and porcine EAs were found when thrombin was present at a concentration of $5 \mathrm{NIHU} / \mathrm{mL}$ blood $(P<.05)$ (Fig $7 A-C)$. Elasticity of the thrombin-induced porcine EAs was higher than that of the thrombin-induced human EAs $(P<.05)$ (Fig $7 C)$. Compared with the counterparts of the bovine and human EAs, thrombin-induced porcine EAs retracted the most (data not shown). Addition of thrombin resulted in increases in $\mathrm{E}_{0 \%-75 \%}$ and elasticity of human EAs $(P<.05)$ (Fig $7 A-C)$. In the absence of thrombin and barium sulfate, the bovine EAs had strain recovery of $30.3 \pm 2.5 \%$, whereas strain recovery in human bloodderived EAs was immeasurable due to their fragile nature. Less EA shrinkage and a significant decrease in elasticity $(P<.05)$ were found in all barium sulfate-containing EAs (Fig $7 F$ ). Due to the immiscibility between barium sulfate and blood, barium sulfate precipitation at the bottom of EAs was observed. However, the accelerated clotting process induced by thrombin improved the homogeneity of barium sulfate dispersion. The structure of the barium sulfate-impregnated EAs is shown in Fig $6 E,-F$. The area under the S-S curve from initial to 95\% strain increased with the addition of barium sulfate, indicating the EAs became tougher (Fig 7G).

A series of comparisons between the different types of EAs and emboli obtained from patients showed recalcified porcine EAs and thrombin-induced ( $5 \mathrm{NIHU} / \mathrm{mL}$ blood) human EAs 

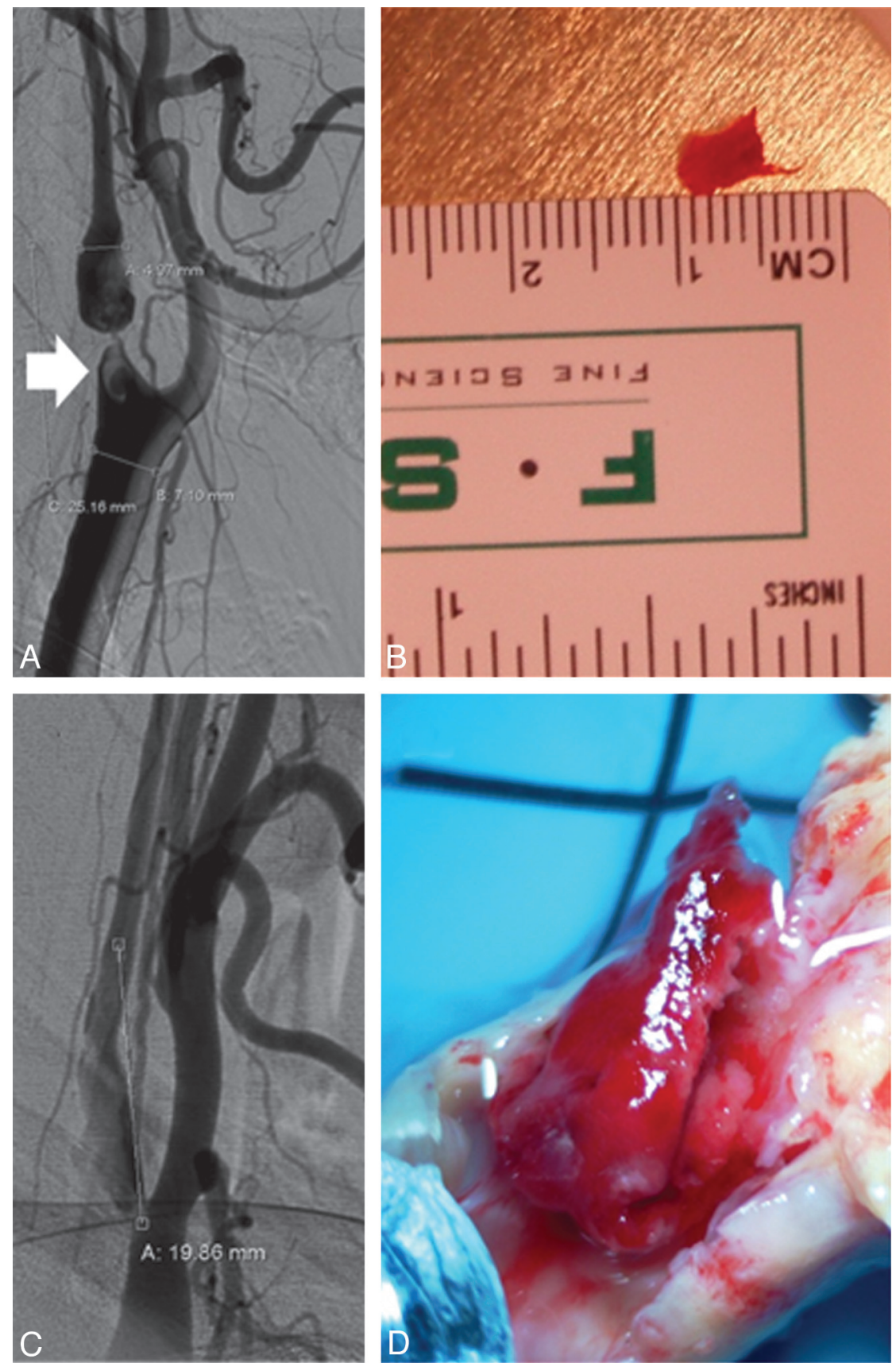

Fig 2. A 65-year-old man (patient 5) presents with an AIS due to MCA occlusion and associated right ICA origin segmental stenosis (>90\%) with intraluminal clot (A, arrow). The clot $(B)$ is removed by using the aspiration device for characterization. A 53-year-old man (patient 11) with transient ischemic attacks has a clot located at the carotid bifurcation (C). After CEA, red thrombus $(D)$ is collected for mechanical and structural analyses.

are similar to cerebral emboli retrieved from the AIS patients in terms of stiffness and elasticity $(P>.05)$. The thrombininduced bovine EAs (2.5 and $5 \mathrm{NIHU} / \mathrm{mL}$ blood) and thrombin-induced porcine EAs ( $5 \mathrm{NIHU} / \mathrm{mL}$ blood) best represent the plaque material collected at CEA.

\section{Discussion}

Embolus characteristics in terms of mechanical properties, composition, and structure are important in the endovascular treatment of AIS. Recently, manual elongation tests were performed by using forceps to stretch EAs in an effort to understand their mechanics. ${ }^{4}$ In this present work, stiffness and elasticity of the in vitro EAs were quantitatively measured and compared with the clinical thromboemboli that they are intended to represent.

From the human specimens, we were able to ascertain a large variation of bulk mechanical properties associated with heterogeneous composition. Some of this variability was inherent to the sample population, which included both emboli retrieved from patients in the acute phase of the stroke and material from CEA. Most CEA specimens were attached to the lumen of the plaque and represent potential stroke-inducing emboli. The other CEA specimens were located immediately under the fibrous cap, and though it is possible that these materials could lead to an artery-to-artery embolism after plaque rupture, we assume that this is an unlikely event. The 

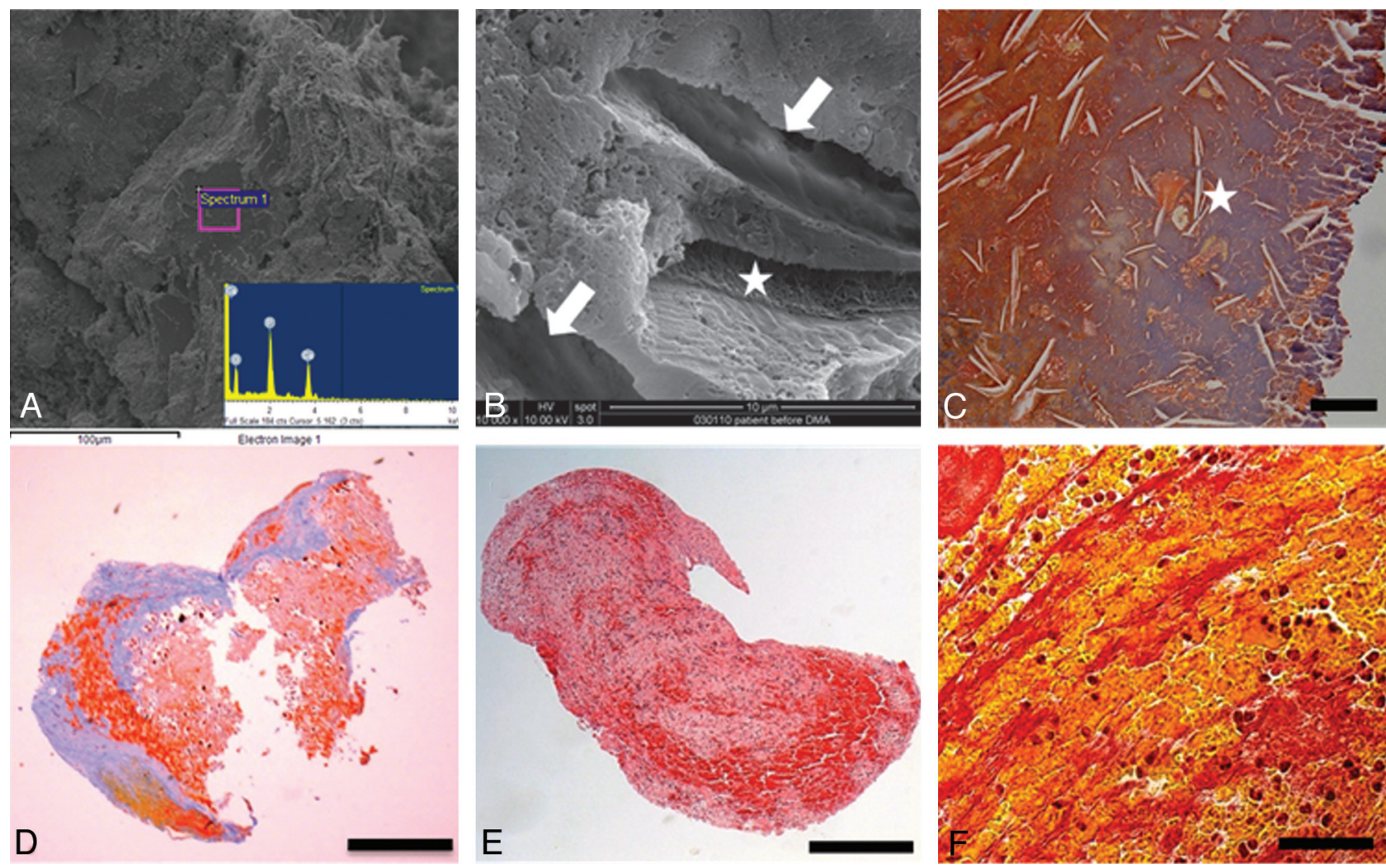

Fig 3. Morphologic features and composition of a variety of emboli from patients. $A$, Calcified embolus calcium-phosphate apatite is detected by the EDS scan (inset). $B$, An aged embolus has a compact structure with fissures that were occupied by the cholesterol crystals (arrows) and fibrin at the edge of the specimen (star). The SEM findings are related to the MSB result $(\times 20$; bar $=100 \mu \mathrm{m})$ shown in C. D. MSB results show that a red embolus is mainly composed of fibrin and erythrocytes (old fibrin in blue, erythrocytes in yellow, and erythrocyte-fibrin mixture in red) $(\times 2$, bar $=1 \mathrm{~mm})$. E, Photomicrograph of a red embolus retrieved from the stroke patient (H\&E $\times 10$, bar $=200 \mu \mathrm{m})$ reveals that the leukocytes are distributed throughout the embolus. F, At $\times 10$ magnification, erythrocytes and fibrin strands are arranged in a layer-by-layer manner in the red embolus (MSB, bar $=200 \mu \mathrm{m}$ ).

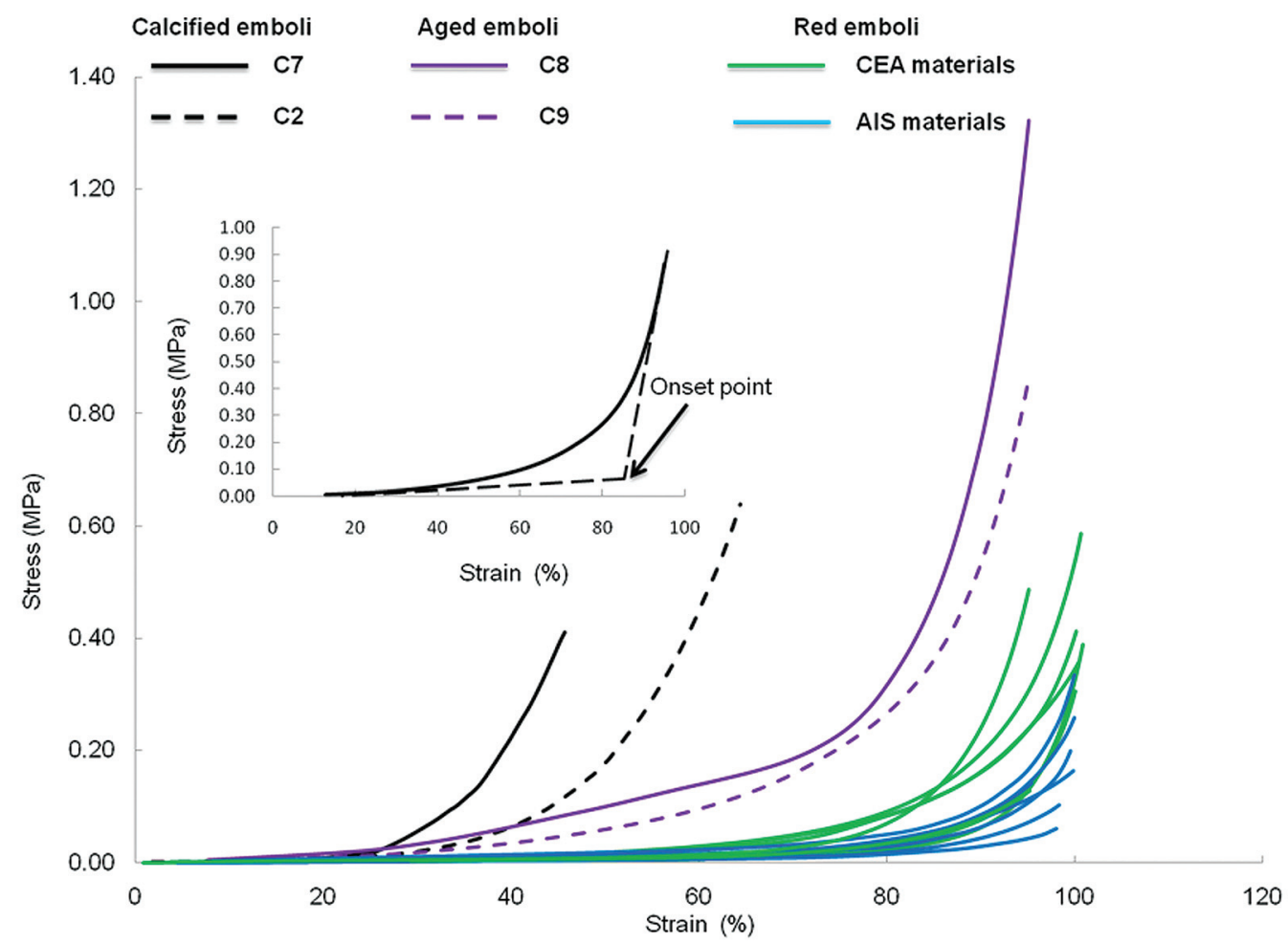

Fig 4. The engineering S-S curves show that at the end of the test, a $15-\mathrm{N}$ force causes a $45.7 \%$ strain to a highly calcified embolus (black solid line) and a $64.4 \%$ strain to a partially calcified embolus (black dashed line). The same compression force results in a higher strain $(>80 \%)$ on the other emboli. 


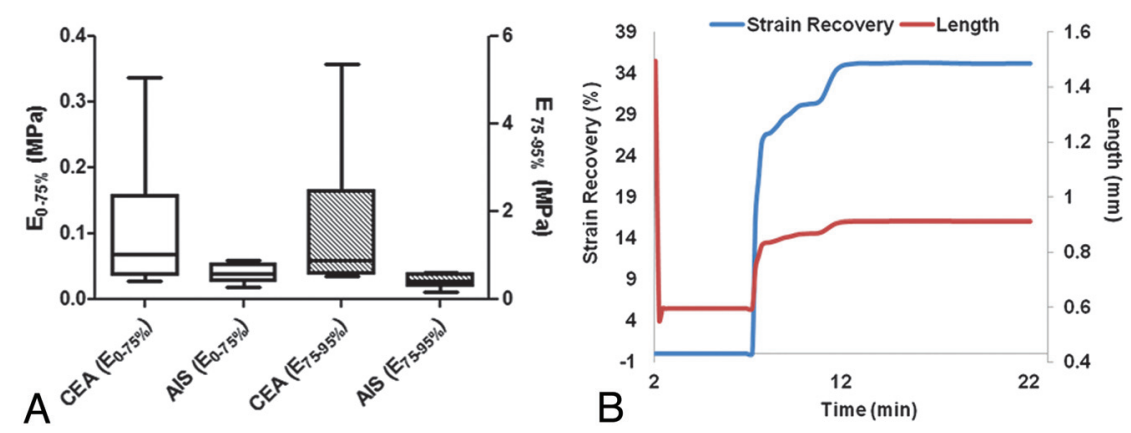

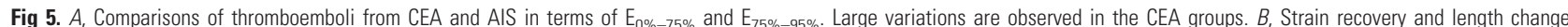
of one of the thromboemboli during the stress relaxation test. The specimen has an initial length of $1.5 \mathrm{~mm}$ and was compressed to $60 \%$ strain for 5 minutes. Increases in length and strain recovery are seen after the load is removed.
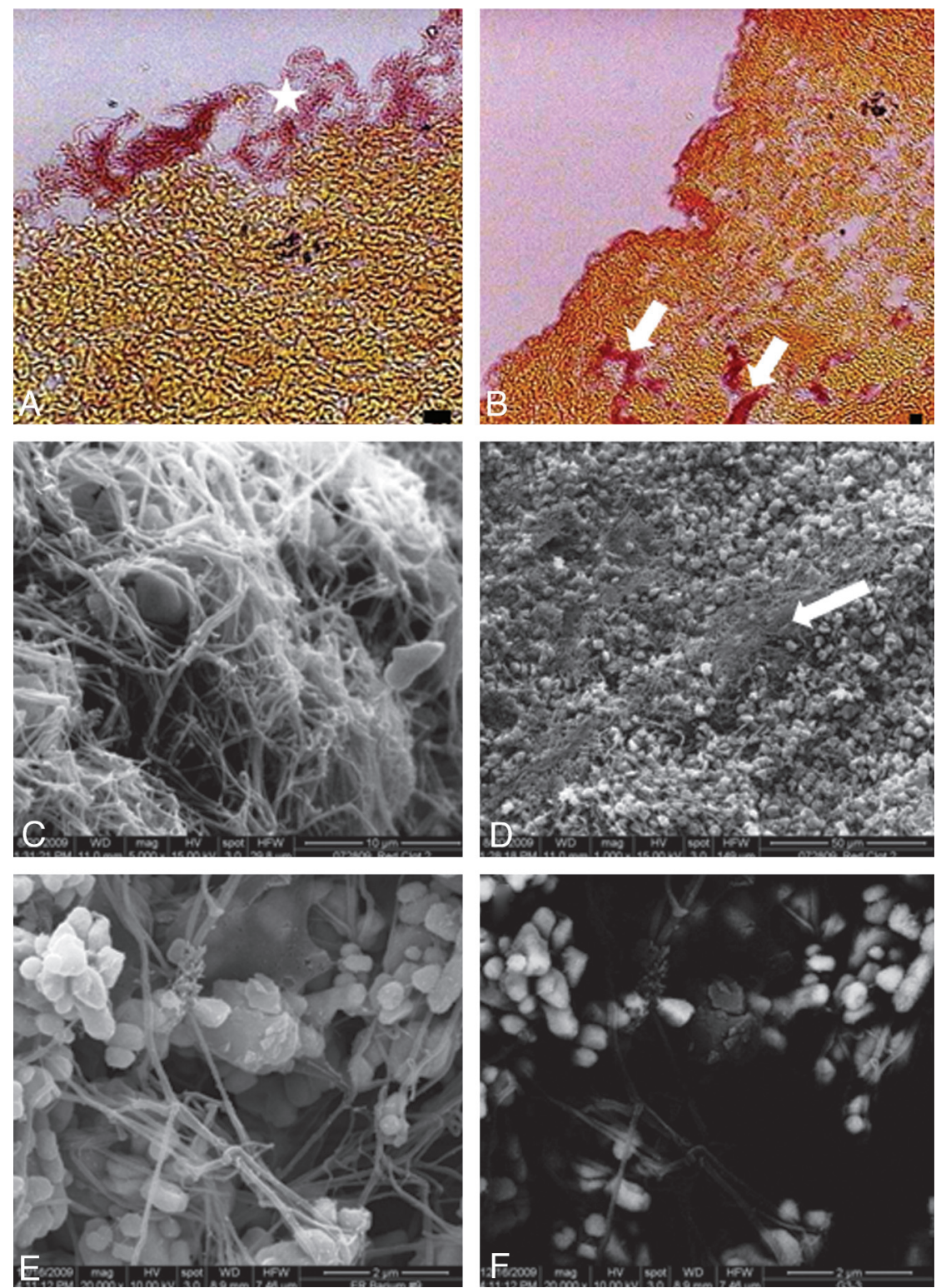

Fig 6. $A$, A layer of fibrin is formed on the surface of the thrombin-induced bovine EA against the silicone tubing (star). MSB staining, bar $=10 \mu \mathrm{m}$. $B$, EA has a homogeneous structure, and is mainly composed of erythrocytes (shown in yellow) with fibrin clumps dispersed in it (arrows, bar $=10 \mu \mathrm{m})$. The corresponding SEM findings are presented in $C(\times 5000)$ and $D$ $(\times 1000)$, respectively. E, Secondary electron image of the bovine EA with barium sulfate $(\times 20000) . F$, Mixed secondary and backscattered electron image of barium sulfate agglomerates $(\times 20000)$. 

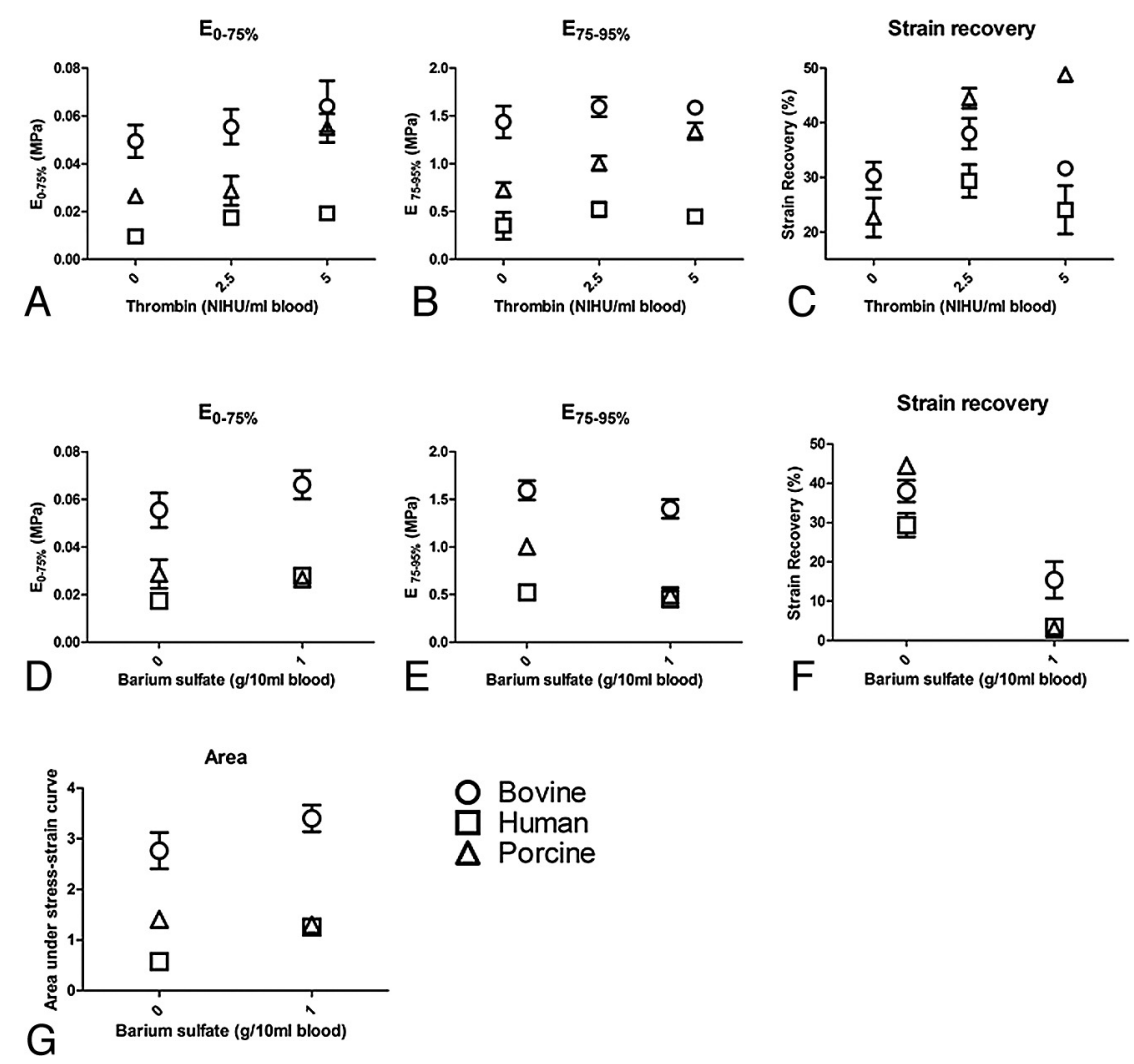

Fig 7. Impact of thrombin and barium sulfate on the average stiffness $\left(E_{0 \%-75 \%}\right.$ and $\left.E_{75 \%-95 \%}\right)$ and elasticity of the bovine, human, and porcine $E A s$ and area under the S-S curve.

dark reddish brown, aged thromboemboli had highly compact structure. These samples lacked elasticity and were prone to fragment (observed during sample preparation for DMA and SEM analyses), which could be associated with the protein degradation of these aged materials. This experience was concordant with the previous findings that aged thrombi provided little resistance to mechanical thrombectomy. ${ }^{25}$ The red thromboemboli retrieved from the AIS patients were soft and elastic. Compared with aged specimens, the red thromboemboli were less likely to fragment. The central challenge to preclinical clot modeling is the fundamental difference between EAs and the human material that they are intended to represent. In the future, to be able to identify which thrombectomy technology is best suited for an embolus of prescribed mechanical properties, it will be necessary to test these devices with a large variety of EAs that span a large range of stiffness and elasticity.

To address this challenge, we prepared EAs by using blood from different species under different clotting conditions that produced variations in stiffness and elasticity. Although these simple EAs have a homogeneous composition and are not structurally representative of the complex human specimens, variations in the bulk mechanical properties were measured and reported. In the development of endovascular recanalization devices in the preclinical setting, the mechanical properties of the EA model alter the device performance. For example, recently an extracranial vascular occlusion model using barium sulfate-doped EAs was used to evaluate the efficacy of an aspiration thrombectomy device. ${ }^{12}$ The applied force from the aspiration system initially elongated the EA but was not sufficient to retrieve or fragment the EAs. This particular study demonstrated that the device does not reliably produce angiographic recanalization in the described model system. However, data reported in the present study show that barium has a significant effect on the properties of the EA, namely, a significant decrease in elasticity. Use of representative clot models may help to improve the predictive capacity of preclinical evaluation of thrombectomy devices.

Overall, this project has quantified the bulk material properties of EAs commonly used in mechanical revascularization research. It is currently not possible with these models to represent the entire diversity of the human specimens that we measured, and this is the focus of our future research. Our study was limited by a unidirectional compression test to explore the material properties of anisotropic specimens. However, the small size of the thromboemboli makes it difficult to measure the hardness and elasticity in different directions. It is important to note that 1 ) the modulus presented in this study is only for comparison due to the use of the sandpaper; and 2) unlike polymer and other synthetic materials, the stiffness of the biologic tissue may depend on strain. The elasticity of the calcified thromboemboli was not available in this study. The force required to cause $60 \%$ strain on the calcified thromboemboli exceeded the limitation of our equipment due to the high stiffness of these samples. Despite these limitations, we were able to fully characterize common EAs used in the laboratory and compare them with the thromboemboli they are intended to represent. Precise characterization of EAs is an important element to proper preclinical evaluation of thrombectomy devices. 


\section{Conclusions}

Differences in structure and composition were observed between EAs and human thromboemboli. Recalcified porcine EAs and thrombin-induced human EAs ( 5 NIHU/mL blood) were similar to cerebral thromboemboli retrieved from patients with AIS in terms of stiffness and elasticity. There was no significant variation between the stiffness of the material collected at CEA and thrombin-induced bovine (2.5 and 5 NIHU/mL blood) or porcine (5 NIHU/mL blood) EAs. Addition of barium sulfate for radio-opacity in the EAs dramatically reduces the elasticity. Fully characterized EAs with known mechanical properties are now available for preclinical evaluation of thrombectomy devices.

\section{Acknowledgments}

We are grateful to Andres Schanzer, MD, and Mohammad H. Eslami, MD, for providing atherosclerotic plaques for characterization and to Stanley Tam, MD, for providing the atrial appendage. We also thank Eileen A. Duhamel, NP, and Anna Thors, NP, for dedicated assistance with this study.

Disclosures: A.K.W., Research Support (including provision of equipment or materials): Philips Med, Details: Research support in form of equipment lease and support of research staff and research projects, Consultant: Covidien, Codman JNJ, Boston Scientific, Soteira. Details: Consulting agreement, payment on hourly basis, Ownership Interest: Surpass Med., Details: Major stockholder; J.P.W., Consultant: Boston Biomedical Associates, Details: Serve on a safety review board for clinical trial of an implantable spine device; M.J.G., Research Support (including provision of equipment or materials): NIH, TSI, Boston Scientific, Micrus Endovascular, Codman Neurovascular, Philips Healthcare, Concentric Medical, NIT, Details: NIH-salary support, research support; Industry noted above: Research support on a fee-for-service basis, Consultant: Soteira Inc, Codman Neurovascular, Micrus Endovascular, Details: Engineering consultation on a fee-for-service basis; Other Financial Relationships: Hologic, Details: Spouse is employed at Hologic.

\section{References}

1. Donnan GA, Baron JC, Ma H, et al. Penumbral selection of patients for trials of acute stroke therapy. Lancet Neurol 2009;8:261-69

2. Smith WS, Sung G, Saver J, et al. Mechanical thrombectomy for acute ischemic stroke: final results of the Multi MERCI trial. Stroke 2008;39:1205-12

3. The Penumbra pivotal stroke trial: safety and effectiveness of a new generation of mechanical devices for clot removal in intracranial large vessel occlusive disease. Stroke 2009;40:2761-68

4. Kan I, Yuki I, Murayama Y, et al. A novel method of thrombus preparation for use in a swine model for evaluation of thrombectomy devices. AJNR Am J Neuroradiol 2010;31:1741-43

5. Asakura F, Yilmaz H, Abdo G, et al. Preclinical testing of a new clot-retrieving wire device using polyvinyl alcohol hydrogel vascular models. Neuroradiology 2007;49:243-51

6. Liebig T, Reinartz J, Hannes R, et al. Comparative in vitro study of five mechanical embolectomy systems: effectiveness of clot removal and risk of distal embolization. Neuroradiology 2008;50:43-52

7. Brekenfeld C, Schroth G, El-Koussy M, et al. Mechanical thromboembolectomy for acute ischemic stroke: comparison of the catch thrombectomy device and the Merci Retriever in vivo. Stroke 2008;39:1213-19

8. Muller-Hulsbeck S, Grimm J, Leidt J, et al. Comparison of in vitro effectiveness of mechanical thrombectomy devices. J Vasc Interv Radiol 2001;12:1185-91

9. Salazar GM, Faintuch S, Gladstone SR, et al. In vitro analysis of downstream particulates with mechanical thrombectomy devices: comparison of $20-\mathrm{kHz}$ sonothrombolytic and rotating dispersion wire systems. J Vasc Interv Radiol 2009;20:634-39

10. Krueger K, Deissler P, Coburger S, et al. How thrombus model impacts the in vitro study of interventional thrombectomy procedures. Invest Radiol 2004;39:641-48

11. Gralla J, Schroth G, Remonda L, et al. A dedicated animal model for mechanical thrombectomy in acute stroke. AJNR Am J Neuroradiol 2006;27:1357-61

12. Gralla J, Schroth G, Remonda L, et al. Mechanical thrombectomy for acute ischemic stroke: thrombus-device interaction, efficiency, and complications in vivo. Stroke 2006;37:3019-24

13. Hong AS, Chae JS, Dubin SB, et al. Ultrasonic clot disruption: an in vitro study. Am Heart J 1990;120:418-22

14. Gersh KC, Nagaswami C, Weisel JW. Fibrin network structure and clot mechanical properties are altered by incorporation of erythrocytes. Thromb Haemost 2009;102:1169-75

15. Di Stasio E, Nagaswami C, Weisel JW, et al. $\mathrm{Cl}^{-}$regulates the structure of the fibrin clot. Biophys J 1998;75:1973-79

16. Ryan EA, Mockros LF, Weisel JW, et al. Structural origins of fibrin clot rheology. Biophys J 1999;77:2813-26

17. Stormorken H. Species differences of clotting factors in ox, dog, horse, and man; thrombin and fibrinogen. Acta Physiol Scand 1957;40:167-81

18. Gentry PA. Comparative aspects of blood coagulation. Vet J 2004; 168:238-51

19. Marder VJ, Chute DJ, Starkman S, et al. Analysis of thrombi retrieved from cerebral arteries of patients with acute ischemic stroke. Stroke 2006; 7:2086-93

20. Almekhlafi MA, Hu WY, Hill MD, et al. Calcification and endothelialization of thrombi in acute stroke. Ann Neurol 2008;64:344-48

21. French JE. The structure of natural and experimental thrombi. Ann R Coll Surg Engl 1965;36:191-200

22. Wechsler LR. Ulceration and carotid artery disease. Stroke 1988;19:650-53

23. Wolf PA, Abbott RD, Kannel WB. Atrial fibrillation as an independent risk factor for stroke: the Framingham Study. Stroke 1991;22:983-88

24. Lendrum AC, Fraser DS, Slidders W, et al. Studies on the character and staining of fibrin. J Clin Pathol 1962;15:401-13

25. Grimm J, Jahnke T, Muhle C, et al. Influence of thrombus age on the mechanical thrombectomy efficacy of the Amplatz thrombectomy device in vitro. Cardiovasc Intervent Radiol 2003;26:265-68 